\title{
Adipokines and parameters of peritoneal membrane transport in newly started peritoneal dialysis patients
}

\author{
Edyta Golembiewska ${ }^{\bowtie}$, Krzysztof Safranow$^{2}$, Kazimierz Ciechanowski', Joanna Bober ${ }^{3}$, \\ Anna Bogacka ${ }^{4}$ and Joanna Stepniewska ${ }^{1}$
}

'Department of Nephrology, Transplantology and Internal Medicine, Pomeranian Medical University, Szczecin, Poland; ${ }^{2}$ Department of Biochemistry and Medical Chemistry, Pomeranian Medical University, Szczecin, Poland; ${ }^{3}$ Department of Medical Chemistry, Pomeranian Medical University, Szczecin, Poland; “Department of Human Nutrition, West Pomeranian University of Technology, Szczecin, Poland

\begin{abstract}
Aim: Adiponectin and leptin are two adipokines playing important roles in the regulation of body weight, appetite, carbohydrate and lipid metabolism. In patients with chronic kidney disease (CKD) adipokines accumulate in serum due to reduced renal clearance. Since adipokines have pleiotropic actions, the adipokine balance may have an impact on peritoneal membrane transport. The aim of this study was to assess whether serum adiponectin and leptin levels were related to peritoneal transport and residual renal function (RRF) in newly started peritoneal dialysis patients. Methods: 25 clinically stable patients, 4 to 6 weeks after the onset of PD, were included in the study. For each patient clinical and laboratory data were reviewed and standard PET test was performed. Serum adiponectin and leptin concentrations were determined and leptin/adiponectin (L/A) ratio was calculated. Results: Serum adiponectin correlated negatively with weight, BMI and glucose concentration. Serum leptin and L/A ratio correlated positively with BMI. Serum adiponectin correlated positively with dialysate to plasma (D/P) creatinine ratio and ultrafiltration in PET test. Serum leptin level and L/A ratio correlated strongly negatively with peritoneal creatinine clearance. Conclusions: Serum adiponectin concentration is positively associated with baseline solute transport. Leptin concentration and L/A ratio are negatively associated with dialysis adequacy in newly started PD patients.
\end{abstract}

Key words: peritoneal dialysis, serum adiponectin, serum leptin, solute transport

Received: 02 August, 2013; revised: 14 October, 2013; accepted: 22 November, 2013; available on-line: 20 December, 2013

\section{INTRODUCTION}

In recent years adipose tissue has been more and more recognized as an endocrine organ involved in many metabolic processes. It is a source of various cytokines, including adiponectin, leptin, tumor necrosis factor-alpha, interleukin-6, resistin, visfatin and other (Więcek et al., 2007).

Adiponectin and leptin are two adipokines which play important roles in the regulation of body weight, appetite, carbohydrate and lipid metabolism. Stimulation of an inflammation and modulation of the immune system has been shown for leptin. Adiponectin, on the other hand, is known of anti-inflammatory and antiatherogenic properties. Low circulating levels of adiponectin are associated with an endothelial dysfunction and a higher risk of cardiovascular complications in the general popu- lation. Plasma leptin/adiponectin ratio (L/A ratio) has been proposed recently as a novel marker for atherosclerosis and as a predictor of cardiovascular events both in population of the patients with diabetes and the peritoneal dialysis (PD) patients as well (Teta et al., 2008; Hara et al., 2011; Satoh et al., 2004; Park et al., 2013).

In patients with chronic kidney disease (CKD) adipokines accumulate in serum what is attributable to reduced renal clearance. Nevertheless, other factors, such as chronic inflammation or nutritional status, may affect adiponectin and leptin levels in CKD patients (Slee, 2012).

In PD glucose-based fluids may influence the activity of adipose tissue and release of cytokines (Park et al., 2008; Lai \& Leung, 2010). Studies in vitro showed that glucose-containing fluids upregulated leptin and downregulated adiponectin production from cultured adipocytes. It has been found that plasma leptin concentrations in PD patients are distinctly higher than the ones observed in haemodialysis patients or non-dialyzed CKD patients (Diez et al., 2005). Compared to leptin, in one cross-sectional study plasma adiponectin levels did not differ between PD and HD patients (Huang et al., 2004). For the reason that adipokines have pleiotropic actions, the adipokine balance may have an impact on peritoneal membrane transport.

The aim of this study was to assess whether serum adiponectin and leptin levels were related to peritoneal transport and residual renal function (RRF) in incident peritoneal dialysis patients.

\section{METHODS}

Patients. Twenty five incident peritoneal dialysis patients at Peritoneal Dialysis Center, Department of Nephrology, Transplantology and Internal Medicine, Szczecin, Poland, were enrolled in the study 4-6 weeks after the dialysis onset. All patients were started on continuous ambulatory peritoneal dialysis (CAPD) using four $2 \mathrm{~L}$ glucose-based dialysates with calcium concentration of $1.25 \mathrm{mmol} / \mathrm{L}$ (Baxter Healthcare). The patients were clinically stable and did not present the signs and symptoms of an overt infection or a malignancy. None of the

e-mail: irys@pum.edu.pl

Abbreviations: ACE, angiotensin converting enzyme; $A R B$, angiotensin II receptor blocker; $\mathrm{BMI}$, body mass index; CAPD, continuous ambulatory peritoneal dialysis; CKD, chronic kidney disease; CRP, Creactive protein; $\mathrm{L} / \mathrm{A}$ ratio, leptin/adiponectin ratio; $\mathrm{PD}$, peritoneal dialysis; RRF, residual renal function; SLE, systemic lupus erythematosus 
Table 1. Characteristics of a study population.

\begin{tabular}{ll}
\hline Age (years) & $47(32-63)$ \\
Gender (males/females) (\%) & $12 / 13(48 \% / 52 \%)$ \\
Diabetes n (\%) & $1(4 \%)$ \\
BMI (kg/m2) & $25.6(22.5-27.6)$ \\
RRF (mL/min/1.73 m2) & $11.1(6.3-13.2)$ \\
Diuresis (mL/day) & $1800(1100-2600)$ \\
Total weekly Kt/V & $2.85(2.52-3.34)$ \\
Peritoneal Kt/V & $1.18(1.05-1.39)$ \\
Peritoneal creatinine clearance (L/week/1.73m2) & $37(31.8-41.2)$ \\
Dialysate to plasma (D/P) ratio at 4 hours in PET & $0.63(0.58-0.72)$ \\
D/D0 glucose ratio in dialysate in PET & $0.43(0.37-0.48)$ \\
UF (mL) & $300(200-400)$ \\
Use of ACE inhibitors (\%) & $6(24 \%)$ \\
Fasting serum glucose (mg/dL) & $89(85-104)$ \\
HbA1c (\%) & $5.4(5.00-5.75)$ \\
Serum total cholesterol (mg/dL) & $207(177-258)$ \\
Serum triglycerides (mg/dL) & $180(121-211)$ \\
Serum albumin (g/L) & $39(37-41)$ \\
Serum C-reactive protein (mg/L) & $4.2(0.9-11.9)$ \\
Serum urea (mg/dL) & $80(73-98)$ \\
Serum creatinine (mg/dL) & $4.4(3.6-6.4)$ \\
Serum adiponectin ( $\mu \mathrm{g} / \mathrm{mL})$ & $18.2(10-26.2)$ \\
Serum leptin (ng/mL) & $16.3(5.5-48.9)$ \\
L/A ratio & $0.89(0.3-3.0)$ \\
Hb (mmol/L) & $7.3(6.8-7.6)$ \\
Hematocrit (L/L) & $0.34(0.33-0.37)$ \\
\hline
\end{tabular}

RRF, residual renal function evaluated using creatinine clearance determined by the UV/P formula: RRF $=24 \mathrm{~h}$ urine creatinine concentration $(\mathrm{mg} / \mathrm{mL}) \times 24 \mathrm{~h}$ urine output $(\mathrm{mL}) /$ serum creatinine $(\mathrm{mg} / \mathrm{mL}) \times 1440$; UF -ultrafiltration in $\mathrm{mL}$. Data are given as median (lower quartile - upper quartile).

patients had peritonitis at the time of the study or during the 4 weeks preceding the study. All patients gave written informed consent. The study was approved by the local Bioethics Committee.

The charts of these patients were reviewed to assess age, gender, weight (measured while dialysate was drained out), body mass index (BMI), type of nephropathy, presence of diabetes, residual renal function (RRF), use of angiotensin converting enzyme (ACE) inhibitors and angiotensin II receptor blockers (ARB).

The main characteristics of the study group are presented in Table 1. The causes of ESRD were as follows: chronic glomerulonephritis (9), diabetes (1), SLE (2), rheumatoid arthritis (1), hypertension (1), chronic pyelonephritis (1), congenital urinary tract abnormality (1), plasmocytoma (1), unknown (8). The study group consisted of 'early start' patients with relatively high residual renal function and total $\mathrm{Kt} / \mathrm{V}$.

Procedure. A fasting blood sample was collected from each patient. Measurements of serum urea, creatinine, glucose, HbA1c, total cholesterol, triglycerides, albumin, CRP were performed using routine laboratory techniques on Architect c8000, Abbott.

Serum adiponectin levels were determined using a commercially available human enzyme immunoassay kit (Quantikine Human Adiponectin, R\&D systems, Minneapolis, MN, USA). The coefficients of variation for intra- and inter-assay precision were $<5 \%$ and $7 \%$, respectively. Serum leptin levels were determined using a commercially available human enzyme immunoassay kit
(Quantikine Human Leptin, R\&D systems, Minneapolis, MN, USA). The coefficients of variation for intra- and inter-assay precision were $<4 \%$ and $6 \%$, respectively. After that, leptin to adiponectin ratio was calculated.

PET test was performed with $2.27 \%$ glucose dialysate as a standard. Two dialysate samples we obtained immediately after completion of the fluid instillation and after 4 hours. Blood samples were obtained 2 hours after dialysate instillation. D/P for creatinine and D/D0 for glucose were calculated. 24-hour effluent and urine were also collected for measurement of $\mathrm{Kt} / \mathrm{V}$ and weekly peritoneal creatinine clearance.

Statistical analysis. Spearman's rank correlation coefficient (Rs) was used to measure associations between quantitative variables. MannWhitney's test was used to compare values between groups. General linear model (GLM) was used for multivariate analysis, with prior logarithm transformation of variables with nonnormal distribution. Quantitative data were presented as median (lower quartile - upper quartile). $P$-value $<0.05$ was considered statistically significant.

\section{RESULTS}

\section{Relationship between adipokines and clinical features}

Serum adiponectin concentrations were significantly higher in women than in men [26.2 (18.3-38.4) vs. 10.4 (8.4-17.2) $\mu \mathrm{g} / \mathrm{mL}, p=0.0007]$, but no correlation with age was detected.

Significant correlations between adipokines and clinical parameters are presented in Table 2.

Leptin level correlated neither with adiponectin level nor with RRF. Neither adiponectin nor leptin level correlated with $\mathrm{Kt} / \mathrm{V}$ values.

No significant relationships between adipokines' concentrations and CRP were observed.

Serum adiponectin level was significantly higher in the subgroup of patients using ACE inhibitors in compare to the subgroup of patients not receiving ACE inhibitors [33.9 (24.8-46.8) vs. $16.2(9.4-21.8) \mu \mathrm{g} / \mathrm{mL}, p=0.01]$.

\section{Relationship between adipokines and peritoneal transport}

The associations of serum adiponectin and leptin levels with parameters of peritoneal dialysis in patients at onset of PD treatment are presented in Fig. 1.

When the group was divided into 2 subgroups according to the $\mathrm{D} / \mathrm{P}$ creatinine cut-off value 0.65 (fast/ fast average - $\mathrm{n}=11$ and slow/slow average transporters - $n=14)$ in spite of significant correlation between $\mathrm{D} / \mathrm{P}$ creatinine and adiponectin level we did not find significant differences in adipokines' levels between these 2 subgroups (for adiponectin $p=0.34$, for leptin $p=0.65$ ).

Peritoneal creatinine clearance and peritoneal $\mathrm{Kt} / \mathrm{V}$ correlated significantly with serum leptin level $(\mathrm{Rs}=-0.52$, $p<0.01$, Rs $=-0.51, p<0.01$, respectively) and $\mathrm{L} / \mathrm{A}$ ratio (Rs $=-0.68, p<0.001$, Rs $=-0.58, p<0.01$, respectively). No significant correlations between adiponectin or leptin level and other examined parameters were observed.

Multivariate analysis with serum leptin concentration logarithm as dependent variable and peritoneal creati- 
A

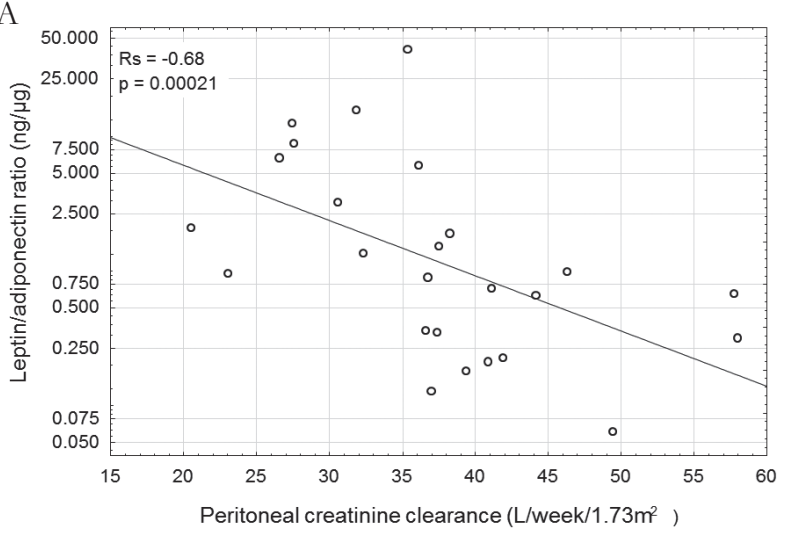

C

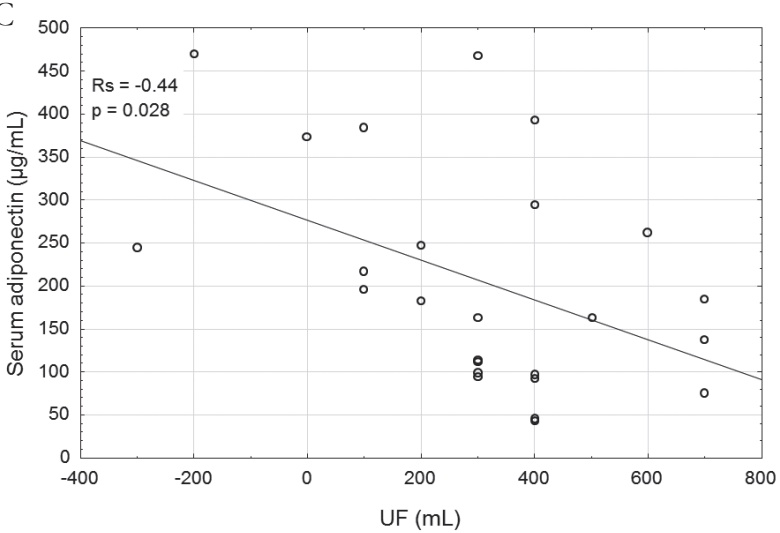

E

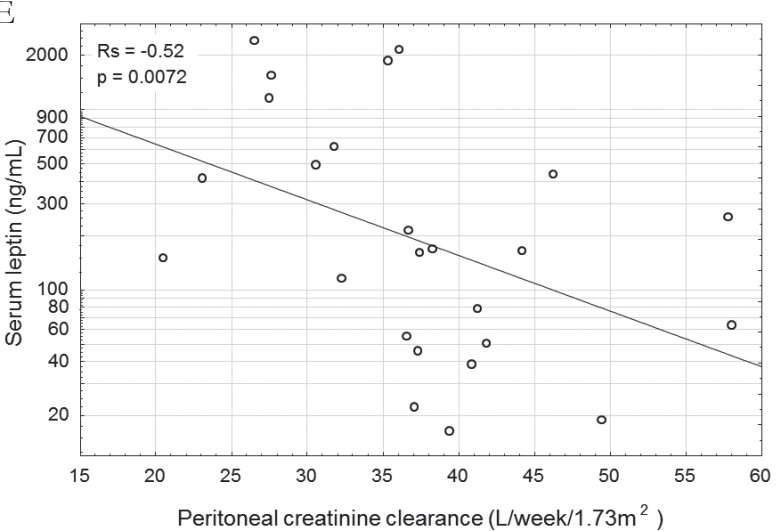

B

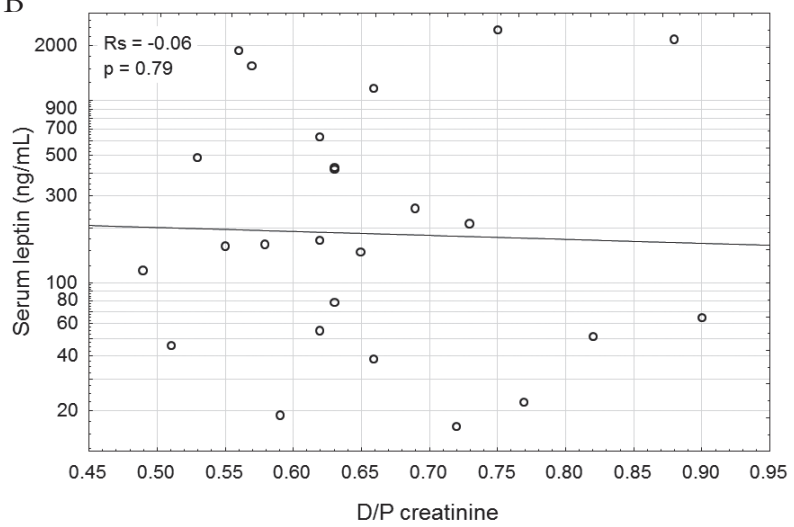

D

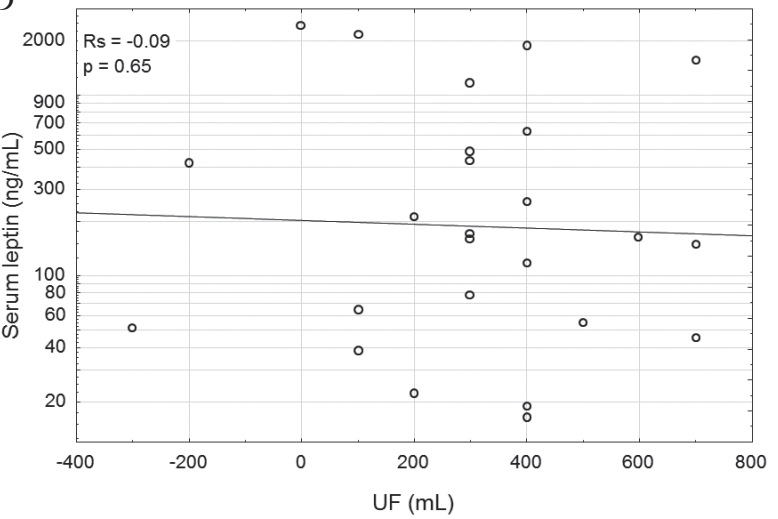

F

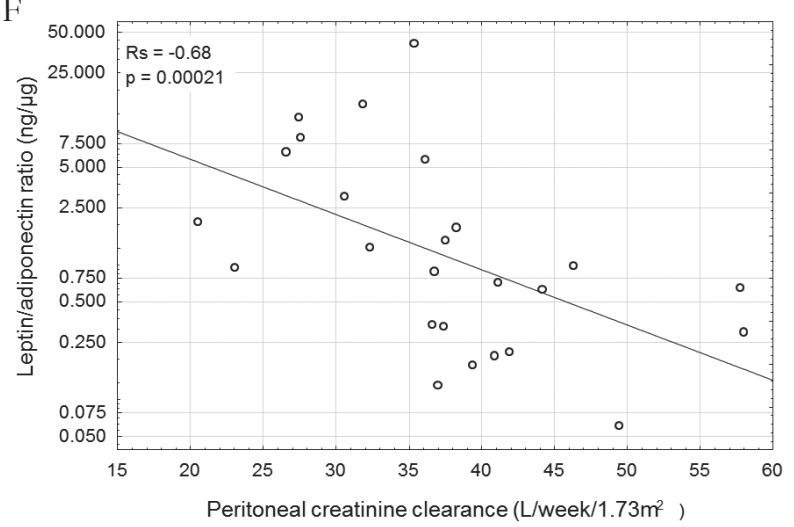

Figure 1A-F. Associations of serum adiponectin and leptin levels with parameters of peritoneal dialysis in patients at onset of PD. Spearman Rs values are presented. Correlations between D/P creatinine and serum adiponectin (A) and serum leptin (B), correlations between UF and serum adiponectin (C) and serum leptin (D), peritoneal creatinine clearance and serum leptin (E) and L/A ratio (F).

nine clearance, BMI and gender as independent variables showed that low peritoneal creatinine clearance $(\beta=$ $-0.32, p=0.012)$, high BMI $(\beta=+0.73, p=0.00001)$ and female gender $(\beta=+0.73, p=0.000005)$ are independent determinants of a high leptin concentration in patients at the onset of PD. Similar model for the logarithm of $\mathrm{L} / \mathrm{A}$ ratio showed also that low peritoneal creatinine clearance $(\beta=-0.35, p=0.014)$, high BMI $(\beta=+0.73$, $p=0.00005)$ and female gender $(\beta=+0.44, p=0.0036)$ are independent predictors of the high $\mathrm{L} / \mathrm{A}$. Only female gender $(\beta=+0.54, p=0.0067)$, excluding peritoneal creatinine clearance $(p=0.42)$ or BMI $(p=0.46)$, was an independent predictor of a high serum adiponectin concentration logarithm. Multivariate models adjusted for BMI and gender did not show association of RRF with leptin, adiponectin or $\mathrm{L} / \mathrm{A}$ ratio $(p>0.4)$.

\section{DISCUSSION}

Adipose tissue was recognized in the last decade as an important source of hormones. The levels of adipokines such as adiponectin and leptin are related to metabolic disturbances. However, these two adipokines show also distinct cytokine properties and take part in the inflammatory processes.

The associations between adiponectin level and the components of the insulin-resistance syndrome (including BMI and glucose level) have been observed in both general population and patients with chronic kidney disease (Guebre-Egziabher et al., 2005; Tsao et al., 2008; Ramos et al., 2009; Lee et al., 2011; An et al., 2011; Eglit et al., 2012). In subjects with metabolic syndrome, patients with type 2 diabetes or coronary artery disease 
Table 2. Significant correlations between adipokines and clinical parameters. Spearman Rs values are presented.

\begin{tabular}{llll}
\hline Correlated parameters & Serum adiponectin & Serum leptin & L/A ratio \\
\hline Weight & $-0.45^{*}$ & 0.28 & $0.43^{*}$ \\
BMI & $-0.40^{*}$ & $0.51^{* *}$ & $0.64^{* * *}$ \\
Glucose & $-0.53^{* *}$ & -0.20 & 0.04 \\
Triglycerides & -0.33 & 0.29 & $0.42^{*}$ \\
RRF & $-0.39^{\wedge}$ & 0.29 & $0.39 \wedge$ \\
\hline
\end{tabular}

$p=0.05,{ }^{*} p<0.05,{ }^{* *} p<0.01,{ }^{* * *} p<0.001$

the serum total adiponectin level is reduced (Kacso et al., 2010; Hara et al., 2011). Studies have shown that serum adiponectin levels are elevated in patients with chronic kidney disease what is predominantly attributable to reduced renal clearance. An inverse relationship was found between plasma adiponectin concentration and kidney function assessed by glomerular filtration rate (GFR) in patients with NKF K/DOQI (The National Kidney Foundation Kidney Disease Outcome Quality Initiative) 3-5 CKD (Nanayakkara et al., 2009) and kidney transplant recipients (Adamczak et al., 2007). Guebre-Egziabher et al. showed that although serum adiponectin level increased when renal function was deteriorated in patients with CKD, at the same time its level was still more significantly influenced by BMI (Guebre-Egziabher et al., 2005). A number of studies (Ramos et al., 2009; Hara et al., 2011; Eglit et al., 2012) showed significant positive association of adiponectin with female gender in the population of patients with normal kidney function and with CKD, both on conservative treatment or those undergoing hemodialysis.

In healthy subjects high adiponectin level appears to be protective against cardiovascular disease. Different mechanisms were proposed for anti-atherogenic and anti-inflammatory actions of adiponectin, such as suppression of TNF-alpha production by macrophages, suppression of superoxide generation, inhibition of the biological actions of growth factors and others (Wieccek et al., 2007). Recently, high plasma leptin/adiponectin ratio (L/A ratio) has been proposed as a novel marker for atherosclerosis. In the population of patients with diabetes $\mathrm{L} / \mathrm{A}$ ratio is related to carotid intima-media thickness (Kotani et al., 2005). In the population of PD patients $\mathrm{L} / \mathrm{A}$ ratio is markedly elevated, what may have implications for patient outcomes (Teta et al., 2008; Park et al., 2013).

Our study showed that in the population of newly started PD patients with relatively high RRF, serum adipokine levels was still strongly correlated with patients' weight and BMI. Although serum adiponectin and leptin levels were not significantly related to triglycerides level, the correlation between L/A ratio and triglycerides achieved statistical significance. Correlation between serum adipokine level and BMI was observed in other studies in PD population (Tsao et al., 2008; An et al., 2011). Positive correlation between L/A ratio and tryglicerydes was recently found in Park study (Park et al., 2013).

Our study showed borderline negative correlation between RRF and serum adiponectin concentration $(p=0.05)$. Nevertheless, multivariate analysis adjusted for gender and BMI disclosed no significant relationship between kidney function and adiponectin level. In the study by Huang et al. (Huang et al., 2004) in 104 PD patients with a mean dialysis duration of $30.3 \pm 37.0$ months serum adiponectin level was inversely related to the glo- merular filtration rate $(\mathrm{r}=-0.313, p=0.001)$. In this study total or peritoneal weekly creatinine clearance, fourth hour of the dialysate to plasma creatinine ratio and the ultrafiltration did not predict adiponectin levels. Alike to other studies the authors concluded that renal clearance had an important influence on serum adiponectin level. The study in renal transplant recipients showed that successful kidney transplantation decreases adiponectin levels, proving that kidney function plays an important role in its elimination (Adamczak et al., 2007).

The interesting finding in the Huang study was that PD patients who absorbed more glucose had higher serum adiponectin levels (Huang et al., 2004). When patients were divided into the groups of high and low transporters according to PET results, the lower transporters had lower adiponectin levels. Our study illustrated significant positive relationship between dialysate to plasma creatinine ratio and serum adiponectin level. However, when the group was divided into fast and slow transporters the difference in adiponectin level was not statistically significant. This discrepancy indicates that the analysis of the correlations provides higher statistical power than dividing a group into 2 very small subgroups using arbitrary criterion $(\mathrm{D} / \mathrm{P}$ creatinine = $0.65)$. Our study did not show relationship between D/ D0 ratio and adiponectin level, although we observed significant negative relationship between ultrafitration achieved in PET and adiponectin level. We observed in our study significant negative relationship between glucose and adiponectin level. It has already been described that adiponectin is an insulin sensitizer, therefore it is possible that it facilitates glucose metabolism and enhances glucose uptake from dialysate. Such negative correlation between adiponectin and glucose has been observed also in hemodialysis patients. On the other hand, Lee et al. performed a study (Lee et al., 2010) on the influence of clinical features, RRF and dialysis regimen on inflammatory cytokines and adipokines in PD patients. When the patients were divided into the groups of high and low peritoneal transport rate, the corresponding adiponectin levels were $24.3 \pm 13.1$ and $21.0 \pm 14.1 \mu \mathrm{g} / \mathrm{mL}$, and there was no statistical difference between these two groups. However it is worth noting that the mean duration of PD therapy was $41.7 \pm 33.1$ months. In contrast, plasma leptin level correlated negatively with adiponectin level and positively with logarithmically transformed CRP level.

In Tsao et al. study on 60 nondiabetic CAPD patients the authors observed a positive trend for correlation between plasma adiponectin level and the ratio of dialysate to plasma creatinine concentration (Pearson $r=0.246$, $p=0.06$ ) (Tsao et al., 2008). Moreover, the study was performed in patients with mean duration of PD treatment was 24.4 \pm 26.6 months. To the best of our knowledge, we are the first to report significant correlation between serum adiponectin level and dialysate to plasma creatinine ratio at 4 hours in newly started PD patients.

Leptin plays key role in appetite suppression and inflammation. Owning the fact that leptin is freely filtered at the glomerulus and degraded in renal tubules, the levels of leptin are higher in CKD, HD and PD patients compared to the healthy subjects. Nanayakkara et al. presented negative linear association between eGFR and plasma leptin in patients with CKD (Nanayakkara et al., 2009). It has been reported that the start of PD treatment is associated with a marked increase in serum leptin levels (Kim et al., 1999; Teta et al., 2008). What is 
interesting is that leptin can be eliminated by PD. However, the removal has little effect on serum leptin level. Hyperleptinemia that was observed during the first months of PD was attributable to glucose load which induces hyperinsulinemia (Teta et al., 2008).

In our study leptin was positively correlated with BMI, but not with RRF. Some studies show that serum leptin is related to inflammatory factors such as CRP (Mak et al., 2006). Momeni and Seirafian stated that serum leptin levels were not correlated with $\mathrm{Kt} / \mathrm{V}$ and number of peritoneal exchanges per day (Momeni et al., 2011). Similarly to Momeni study (Momeni et al., 2011) we did not observe significant relationship between total $\mathrm{Kt} / \mathrm{V}$ and serum leptin level. It might be an interesting finding in our study that serum leptin level was strongly inversely correlated with peritoneal creatinine clearance. Even stronger correlation was observed between peritoneal creatinine clearance and $\mathrm{L} / \mathrm{A}$ ratio. The possible impact of serum adipokine baseline level, L/A ratio and dialysis adequacy on patient's outcome requires prospective studies.

Our study was not designed to examine the influence of ACE inhibition on serum adiponectin levels. However, the results gave substance to recon that patients receiving ACEI had significantly higher levels of serum adiponectin. To the best of the knowledge of the authors, this is the first such an observation in PD patients. Nonetheless, taking into account small number of patients receiving ACEI $(n=6)$, the results need to be considered with caution. The association of ACE inhibition with an increase in plasma adiponectin was found in patients with essential hypertension (Furuhashi et al., 2003). According to Huang's study in patients undergoing $\mathrm{HD}$ or chronic PD treatment, renin-angiotensin (RAS) blockade did not affect adiponectin levels (Huang et al., 2004). The possible implications of RAS blockade on adiponectin levels and outcome in PD patients require longitudinal studies.

\section{PRACTICAL APPLICATION}

We believe that we are the first to report that serum adiponectin level is positively correlated with baseline solute transport in newly started PD patients. We have observed also in these patients that serum leptin level is inversely related to peritoneal creatinine clearance. Serial measurements in the longitudinal observation of patients on PD therapy would undoubtedly strengthen the relevance of our study results. As adiponectin and leptin are more and more regarded as cytokines with inflammatory properties, their precise mechanism of action with possible influence on peritoneal membrane properties and patient's long-term outcome requires further prospective studies.

\section{REFERENCES}

Adamczak M, Szotowska M, Chudek J, Karkoszka H, Cierpka L, Więcek A (2007) Plasma adiponectin concentration in patients after successful kidney transplantation - a single-center, observational study. Clin Nephrol 67: 381-390.

An WS, Son YK, Kim SE Kim KH, Bae HR, Lee S, Park Y, Kim HJ, Vaziri ND (2011) Association of adiponectin and leptin with serum lipids and erythrocyte omega-3 and omega- 6 fatty acids in dialysis patients. Clin Nephrol 75: 195-203.

Diez JJ, Iglesias P, Fernandez-Reyes MJ, Aguilera A, Bajo MA, Alvarez-Fidalgo P, Codoceo R, Selgas R (2005) Serum concentrations of leptin, adiponectin and resistin, and their relationship with cardiovascular disease in patients with end-stage renal disease. Clin Endocrinol 62: 242-249.
Eglit T, Lember M, Ringmets I, Rajasalu T (2012) Gender differences in serum high-molecular-weight (HMW) adiponectin levels in metabolic syndrome. Eur J Endocrinol doi: 10.1530/EJE-12-0688.

Furuhashi M, Ura N, Higashiura K, Murakami H, Tanaka M, Moniwa N, Yoshida D, Shimamoto K (2003) Blockade of the renin-angiotensin system increases adiponectin concentrations in patients with essential hypertension. Hypertension 42: 76-81.

Guebre-Egziabher F, Bernhard J, Funahashi T, Hadj-Aissa A, Fouque $\mathrm{D}$ (2005). Adiponectin in chronic kidney disease is related more to metabolic disturbances than to decline in renal function. Nephrol Dial Transplant 20: 129-134.

Hara K, Uchida T, Takebayashi K, Sakai Y, Inoue T, Inukai T, Takayanagi K, Aso Y (2011) Determinantns of serum high molecular weight (HMW) adiponectin levels in patients with coronary artery disease: associations with cardio-renal-anemia syndrome. Intern Med 50: 2953-2960.

Huang JW, Yen CJ, Chiang HW, Hung KY, Tsai TJ, Wu KD (2004). Adiponectin in peritoneal dialysis patients: a comparison with hemodialysis patients and subjects with normal renal function. $A m J$ Kid Dis 43: 1047-1055.

Kacso IM, Lenghel A, Patiu IM, Racasan S, Orasan R, Rusu CC, Moldovan D, Bondor CI, Nita C, Hancu ND, Caprioara MG (2010) Prognostic value of plasma adiponectin in type 2 diabetic dialysis patients. Therapeutics, Pharmacology and Clinical Toxicology 14: 205-211.

Kim DJ, Oh DJ, Kim B, Lim YH, Kang WH, Lee BH, Lee SK, Huh W, Kim SE, Lee MK, Kang SA, Oh HY (1999) The effect of continuous ambulatory peritoneal dialysis on change in serum leptin. Perit Dial Int 19: S172-S175.

Kotani K, Sakane N, Saiga K, Kurozawa Y (2005). Leptin: adiponectin ratio as an atherogenic index in patients with type 2 diabetes: relationship of the index to carotid intima-media thickness. Diabetologia 48: 2684-2686.

Lai KN, Leung JCK (2010). Peritoneal adipocytes and their role in inflammation during peritoneal dialysis. Mediators Inflamm doi: 10.1155/2010/495416.

Lee CT, Ng HY, Hsu CY, Tsai YC, Yang YK, Chen TC, Chiou T'T, Kuo CC, Lee WC, Hsu KT (2010) Proinflammatory cytokines, hepatocyte growth factor and adipokines in peritoneal dialysis patients. Artif Organs 34: E222-E229.

Lee YJ, Cho S, Kim SR (2011). The association between serum adiponectin levels and nutritional status of hemodialysis patients. Ren Fail 33: 506-511.

Mak RH, Cheung W, Cone RD, Marks DL (2006). Leptin and inflammation-associated cachexia in chronic kidney disease. Kid Int 69: 794-797.

Momeni A, Seirafian S (2011) Relationship between serum leptin level and peritonitis in CAPD patients. Nephro-Urol Mon 3: 272-275.

Nanayakkara PWB, Le Poole CY, Fouque D C, van Guldener, Stehouwer CDA, Smulders YM, van Ittersum FJ, Siegert CEH, Drai J, Kostense PJ, ter Wee PM (2009) Plasma adiponectin concentration has an inverse and a non linear association with estimated glomerular filtration rate in patients with K/DOQI 3-5 chronic kidney disease. Clin Nephrol 72: 21-30.

Park JH, Gil HW, Yang JO, Lee EY, Hong SY (2008) Total and high molecular weight adiponectin concentrations in plasma of patients with end-stage renal disease before and after peritoneal dialysis. Nephrology 13: 181-185.

Park JT, Yoo TH, Kim JK, Oh HJ, Kim SJ, Yoo DE, Lee MJ, Shin DH, Han SH, Han DS, Kang SW (2013) Leptin/adiponectin ratio is an independent predictor of mortality in nondiabetic peritoneal dialysis patients. PDI 33: 67-74

Ramos LF, Shintani A, Himmelfarb J, Ikizler TA (2009) Determinants of plasma adiponectin levels in nondiabetic subjects with moderate to severe chronic kidney disease. J Ren Nutr 19: 197-203.

Satoh N, Naruse M, Usui T, Tagami T, Suganami T, Yamada K, Kuzuya H, Shimatsu A, Ogawa Y (2004) Leptin-to-adiponectin ratio as a potential atherogenic index in obese type 2 diabetic patients. Diabetes Care 27: 2488-2490.

Slee AD (2012) Exploring metabolic dysfunction in chronic kidney disease. Nutrition \& Metabolism doi: 10.1186/1743-7075-9-36.

23. Teta D, Maillard M, Halabi G, Burnier M (2008) The leptin/adiponectin ratio: Potential implications for peritoneal dialysis. Kid Int 73: S112-S118.

24. Tsao YT, Hsu YJ, Chu NF, Lai CH, Chiu JS, Lin SH (2008) Association of plasma adiponectin and cardiovascular risk profiles in nondiabetic uremic patients on peritoneal dialysis. $J$ Nephrol 21: $744-752$.

25. Więcek A, Adamczak M, Chudek J (2007) Adiponectin - an adipokine with unique metabolic properties. Nephrol Dial Transplant 22: 981-988. 